\title{
Electrostatic interaction between dissimilar colloids at fluid interfaces
}

\author{
Arghya Majee 7 Timo Schmetzer, and Markus Bient \\ Max-Planck-Institut für Intelligente Systeme, Heisenbergstr. 3, 70569 Stuttgart, Germany and \\ IV. Institut für Theoretische Physik, Universität Stuttgart, Pfaffenwaldring 57, 70569 Stuttgart, Germany
}

\begin{abstract}
The electrostatic interaction between two non-identical, moderately charged colloids situated in close proximity of each other at a fluid interface is studied. By resorting to a well-justified model system, this problem is analytically solved within the framework of linearized Poisson-Boltzmann (PB) density functional theory (DFT). The resulting interaction comprises a surface and a line part, both of which, as functions of the inter-particle separation, show a rich behavior including monotonic as well as non-monotonic variations. In almost all cases, these variations cannot be captured correctly by using the superposition approximation. Moreover, expressions for the surface tensions, the line tensions and the fluid-fluid interfacial tension, which are all independent of the inter-particle separation, are obtained. Our results are expected to be particularly useful for emulsions stabilized by oppositely charged particles.
\end{abstract}

\section{INTRODUCTION}

Interfaces between two immiscible fluids like oil and water are often energetically very expensive, which is quantified by a high interfacial tension. Therefore, systems featuring fluid interfaces try to reduce their free energy by minimizing the interfacial area. When colloids are suspended in either of the fluid phases, such a reduction is easily achieved via entrapment of the particles at the interface [1]: a wellknown phenomenon that forms the basis of any Pickering emulsion [2]. This finds application in diverse fields including biomedicine, cosmetic industry, oil recovery, water purification, anti-reflective coating and so on [3-5]. One major advantage of particle stabilized emulsions is that the use of risky surfactants can be avoided, which is particularly important for cosmetic, food and pharmaceutical applications [6-8]. In the form of colloidosome [9], it can even be used to prepare inorganic protocells [10].

Emulsions can be stabilized by using a single type of particle or by using a mixture of particles that differ in their charge. Over the last two decades, this latter has been shown to be useful at many instances [6-8, 11-14]. For example, intrinsically hydrophilic particles that adsorb weakly to interfaces, can form flocs of increased hydrophobicity when mixed with oppositely charged particles and attach stably to an interface [12]. Sometimes relatively strongly charged particles, when approaching the interface from the aqueous phase, alone cannot attach to oil-water interfaces due to strong repulsive interaction with the image charge formed in presence of the dielectric jump at the interface [15]. Aggregates of oppositely charged particles facilitate adsorption in this case owing to a reduction of the net charge of the aggregate compared to the single particle [13, 14]. Moreover, even for chemically identical interacting particles, specially at short separations, identical charge densities at the two particles are not always guranteed [16].

Due to the steep trapping potential (typically several orders of magnitude larger than the thermal energy $k_{B} T$ ) felt by the

\footnotetext{
* majee@is.mpg.de

$\dagger$ bier@is.mpg.de
}

particles at an interface, their out-of-plane movement is essentially frozen [3]. On the other hand, the in-plane movement and the structure of the resulting monolayer depends sensitively on the inter-particle interactions. Depending upon the system, particle interaction may consist of different contributions like van der Waals, capillary, electrostatic, steric or magnetic interactions. Here we focus on charged colloids and consider exclusively the electrostatic interaction between them. At low particle concentration, this can be described by a simple dipolar interaction where each particle and its asymmetric counter-ion cloud generate a dipole perpendicular to the interface [17, 18]. However, for systems with oppositely charged particles, where the separation distance can be well below a nanometer, or for dense suspensions such a simple picture cannot be applied.

A semi-analytical solution for interaction between nonuniformly but like-charged particles at an air-water interface has been recently provided which is valid at all separations between the particles [19]. An alternate approach, which makes the problem completely analytically solvable, is to use a relatively simple yet reasonable model system by ignoring the particle curvature and treating them as flat plates due to their short separation [20, 21]. Using this model system with two plates, effective electrostatic pair-interaction between identical colloids situated in the close vicinity of each other has been studied analytically both within and beyond the superposition approximation [20]. However, the interaction of two identical colloidal particles is a situation which is typically not met in reality, e.g., due to various kinds of polydispersities generated during the preparation process, and a proper theory describing the interaction between non-identical particles situated close to each other at a fluid interface is not available so far.

Therefore, here we address this problem by using the aforementioned model system of flat plates with a liquid-liquid interface in between; see Fig. 11. Additionally, we discard any deformation of the fluid-fluid interface and consider it to be planar with a $90^{\circ}$ liquid-particle contact angle. This implies that the particles are dipped equally deep into both fluid phases such that the corresponding reduction of the interfacial area is maximal. Similar situations have been encountered in previous experimental studies [22-27] and have been used in theoretical modelling [13, 26-30] of such systems as well. There 
(a)

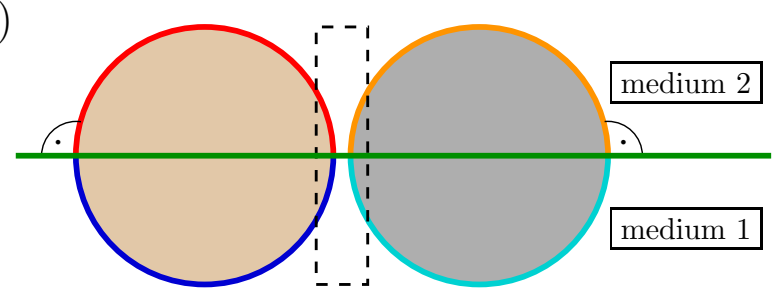

(b)

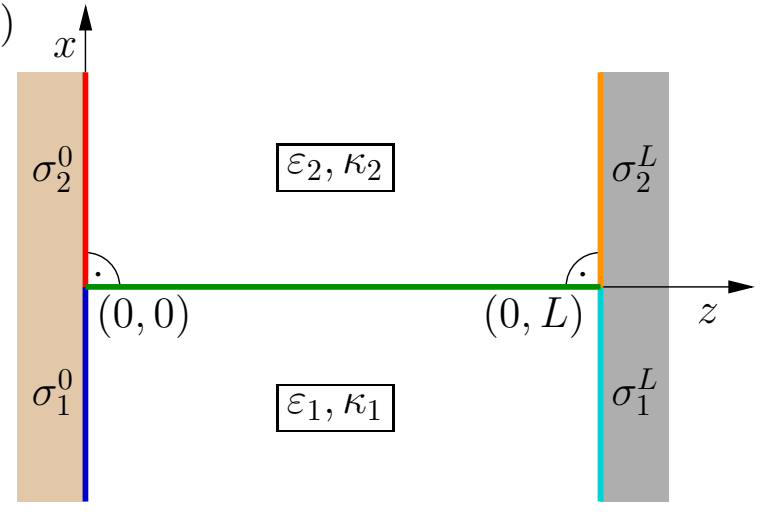

FIG. 1. (a) Sketch of the system under consideration. A pair of non-identical spherical colloids trapped at an oil-water interface (indicated by the horizontal green line) and separated from each other by a distance small compared to their radii. We consider neutralwetting situations corresponding to $90^{\circ}$ liquid-particle contact angles for all particles. (b) Sketch of the simplified system that models the boxed region in panel (a). Due to their short separation, particles are approximated as flat walls positioned at $z=0$ and $L$. The region in between is filled with two immiscible fluids forming an interface at $x=0$. Permittivity and the inverse Debye length of fluid "1" ("2") are given by $\varepsilon_{1}\left(\varepsilon_{2}\right)$ and $\kappa_{1}\left(\kappa_{2}\right)$, respectively. The surfaces carry fixed charge densities $\left(\sigma_{1}^{0}, \sigma_{1}^{L}, \sigma_{2}^{0}\right.$, and $\left.\sigma_{2}^{L}\right)$ which, in general, may not be the same for the two walls and can vary over each wall depending upon the fluid phase it is in contact with.

is strong experimental evidence that charges can be present at the particle-oil interface [31-35]. Accordingly, particles, or the plates of our model system, are assumed to be charged in both the fluid phases. The most salient feature of our system is now the presence of potentially disparate but uniform charge densities on each of the four colloid-fluid contact surfaces.

In this work the model system, as depicted in Fig. 1(b), is analyzed within the framework of classical DFT which allows one to derive the governing equations for the electrostatic potential as well as the boundary conditions and to calculate the resulting effective interactions in a self-consistent manner. First, the electrostatic potential distribution inside the system is calculated once by avoiding and once by using the superposition approximation. In what follows, results obtained in the former case will be denoted as exact results. Next, the interaction between the charged solid surfaces, which can be decomposed into contributions proportional to the areas of the solid surfaces and the lengths of the three-phase contact lines, is calculated using both the exact and the superposition electrostatic potentials. Finally, a comparison of the exact and superposition results is presented for a wide range of system parameters. In addition, our results also provide insight into a particularly striking observation regarding identical particles: all the effective interaction energies of the exact calculation were reported to differ by a factor of 2 from those obtained within the superposition approximation in the asymptotic limit [20]. A conceivable explanation includes the symmetry of the system or the total number of colloidal particles. As will be shown below, the present analysis clearly rules out the former possibility.

\section{FORMALISM}

We confine ourselves to a space filled with two immiscible fluids and bounded by two parallel charged walls placed at $z=0$ and $z=L$ in a Cartesian coordinate system; see Fig. 1(b). The fluid-fluid interface is perpendicular to the walls and situated at $x=0$. The fluid phase spanning the lower (upper) halfspace corresponding to $x<0(x>0)$ is denoted as medium "1" ("2"). The added salt is a binary compound with monovalent components only and the bulk ionic strengths in medium $i \in\{1,2\}$ are $I_{i}$. Since the walls are not identical, surface charge density $\sigma(\mathbf{r})$, in general, is a function of both the spatial coordinates. At contact with medium $i \in\{1,2\}$, the charge densities at the left $(z=0)$ and at the right $(z=L)$ walls are given by $\sigma_{i}^{0}$ and $\sigma_{i}^{L}$, respectively. In general, salt ions, charged surfaces and the fluid-fluid interface influence the structure of the fluids which in turn leads to the number densities $n_{ \pm}(\mathbf{r})$ of the positive and negative ions. However, these structures form on the scale of the bulk correlation length, which usually falls much below the length scale to be considered here. As a result, the fluids are modeled as structureless continuous linear dielectric media characterized by dielectric constants $\varepsilon_{i}=\varepsilon_{r, i} \varepsilon_{0}, i \in\{1,2\}$, where $\varepsilon_{r, i}$ is the relative permittivity of medium $i$ and $\varepsilon_{0}$ is the permittivity of vacuum. Therefore, on sufficiently large length scales, the overall fluid dielectric profile $\varepsilon(\mathbf{r})$ varies step-like at the interface: $\varepsilon(x<0)=\varepsilon_{1}$ and $\varepsilon(x>0)=\varepsilon_{2}$. The charge density $e\left(n_{+}(\mathbf{r})-n_{-}(\mathbf{r})\right)$ in each medium, however, varies on the scale of the respective Debye lengths $\kappa_{i}^{-1}=\sqrt{\varepsilon_{r, i} /\left(8 \pi \ell_{B} I_{i}\right)}$ which set the length scales of our interest and are much larger than the bulk correlation length [36]. Here $\ell_{B}=e^{2} /\left(4 \pi \varepsilon_{0} k_{B} T\right)$ is the vacuum Bjerrum length with the elementary charge $e>0$, the Boltzmann constant $k_{B}$ and the temperature $T$.

Following the standard description at a mean-field level, we treat the ions as point-like objects and ignore their correlation. Considering the bulks of both fluid media as reservoirs with which ions are exchanged the grand canonical density functional corresponding to our system in units of the thermal energy $k_{B} T=1 / \beta$ is then given by

$$
\begin{gathered}
\beta \Omega\left[n_{ \pm}\right]=\int_{V} d^{3} r\left[\sum_{k= \pm} n_{k}(\mathbf{r})\left\{\ln \left(\frac{n_{k}(\mathbf{r})}{\zeta_{k}}\right)-1+\beta V_{k}(\mathbf{r})\right\}\right. \\
\left.+\frac{\beta \mathbf{D}\left(\mathbf{r},\left[n_{ \pm}\right]\right)^{2}}{2 \varepsilon(\mathbf{r})}\right],
\end{gathered}
$$

with the fugacities $\zeta_{ \pm}$of the two ion-species, the electric displacement $\mathbf{D}$, and the solvation free energies $V_{ \pm}(\mathbf{r})$ of the ions. 
The integration volume $V$ is the slab region $(0 \leq z \leq L)$ enclosed by the two walls. The electric displacement $\mathbf{D}$ fulfills Gauss' law

$$
\nabla \cdot \mathbf{D}\left(\mathbf{r},\left[n_{ \pm}\right]\right)=e\left(n_{+}(\mathbf{r})-n_{-}(\mathbf{r})\right)
$$

in the interior of $V$ whereas it is fixed by the given surface charge densities at the walls. The first two terms in the first line of Eq. (1) is the entropic ideal gas contribution of the ions whereas the last term within the curly brackets describes the contribution due to ion-solvent interaction. The last term represents the electric field energy due to the ion distribution and the surface charge densities. First, $\widetilde{\Omega}\left[n_{ \pm}\right]$is obtained from $\Omega\left[n_{ \pm}\right]$by expanding the latter in terms of small deviations of the ion number densities from the bulk ionic strengths and retaining terms up to quadratic order (for details see Chapter II of Ref. [37]). Subsequently $\widetilde{\Omega}\left[n_{ \pm}\right]$is minimized with respect to the ionic density profiles $n_{ \pm}$to obtain the equilibrium profiles

$$
n_{ \pm}^{\mathrm{eq}}(\mathbf{r})=I_{i}\left(1 \mp \beta e\left\{\Psi_{i}(\mathbf{r})-\Psi_{b, i}\right\}\right)
$$

in the two media. Noting the relation $\mathbf{D}=-\varepsilon_{i} \nabla \Psi_{i}$ between the electric displacement $\mathbf{D}$ and the electrostatic potential $\Psi_{i}(\mathbf{r})$ in medium $i \in\{1,2\}$, Eqs. (2) and (3) ultimately lead to the linearized PB or Debye-Hückel (DH) equation

$$
\Delta \Psi_{i}(\mathbf{r})=\kappa_{i}^{2}\left(\Psi_{i}(\mathbf{r})-\Psi_{b, i}\right)
$$

subject to the following boundary conditions: (i) the electrostatic potential must remain finite in the limit $x \rightarrow \pm \infty$, (ii) both the electrostatic potential and the $x$-component of the electric displacement vector should be continuous at the fluid-fluid interface, i.e., $\Psi_{1}\left(x=0^{-}\right)=\Psi_{2}\left(x=0^{+}\right)$and $\varepsilon_{1} \partial_{x} \Psi_{1}\left(x=0^{-}\right)=\varepsilon_{2} \partial_{x} \Psi_{2}\left(x=0^{+}\right)$, and (iii) the $z$-component of the displacement vector should match the charge densities at the surfaces: $\varepsilon_{i} \partial_{z} \Psi_{i}(z=0)=-\sigma_{i}^{0}$ and $\varepsilon_{i} \partial_{z} \Psi_{i}(z=L)=\sigma_{i}^{L}$. These boundary conditions imply the global charge neutrality for our system. $\Psi_{b, i}$ in Eqs. (3) and (4) represents the electrostatic potential in the bulk of the medium $i \in\{1,2\}$. It is constructed such that $\Psi_{b, 1}=0$ and $\Psi_{b, 2}=\Psi_{D}$ where $\Psi_{D}$ originates from an unequal partitioning of ions close to the interface in between the two media and it is known as the Donnan potential or Galvani potential difference between the two fluid phases [38]. If the solvation free energies of the ions are given by $V_{ \pm}(\mathbf{r})=0$ in medium " 1 " and $V_{ \pm}(\mathbf{r})=f_{ \pm}$in medium " 2 ", then the Donnan potential can be written as [20, 37]

$$
\Psi_{D}=-\frac{1}{2 e}\left(f_{+}-f_{-}\right) .
$$

In order to calculate the equilibrium grand potential $\widetilde{\Omega}(L)$ of the system with wall separation $L$ we first rewrite $\widetilde{\Omega}\left[n_{ \pm}\right]$as a functional of the electrostatic potential $\Psi$ by inserting the equilibrium ion density profiles $n_{ \pm}^{\mathrm{eq}}[\Psi]$ considered as functionals of $\Psi$. Then, by plugging the expressions for $\Psi_{i}(x, z)$ obtained by solving Eq. (4), one obtains $\widetilde{\Omega}(L)=\widetilde{\Omega}\left[n_{ \pm}[\Psi]\right]$ which is composed of the following different contributions:

$$
\begin{aligned}
\widetilde{\Omega}(L)= & \sum_{i \in\{1,2\}}\left[\Omega_{b, i} V_{i}+\left(\frac{\gamma_{i}^{0}+\gamma_{i}^{L}}{2}+\omega_{\gamma, i}(L)\right) A_{i}\right] \\
& +\gamma_{1,2} A_{1,2}+\left(\frac{\tau^{0}+\tau^{L}}{2}+\omega_{\tau}(L)\right) \ell,
\end{aligned}
$$

where $\Omega_{b, i}$ is bulk grand potential per volume of medium $i \in\{1,2\} ; V_{i}$ is the volume of medium $i ; \gamma_{i}^{0}$ and $\gamma_{i}^{L}$ are the surface tensions acting between medium " $i$ " and the wall present at $z=0$ and $z=L$, respectively; $\omega_{\gamma, i}(L)$ is the effective interaction energy between surface elements in contact with and acting through medium $i, A_{i}$ is the total area of the two surfaces in contact with medium $i, \gamma_{1,2}$ is the interfacial tension acting between medium 1 and 2; $A_{1,2}$ is the total area of the fluid-fluid interface; $\tau^{0}$ and $\tau^{L}$ are the line tensions acting at the two three-phase contact lines formed at $z=0$ and $z=L$, respectively; and $\omega_{\tau}(L)$ is the effective interaction energy between the contact lines expressed per total length $\ell$ of the two contact lines. Here $\gamma_{i}^{0}, \gamma_{i}^{L}, \tau^{0}$, and $\tau^{L}$ describe the interaction of a single wall with its surrounding fluid(s) and are thus $L$ independent. Therefore, the only $L$-dependent quantities in Eq. (6) are the surface interaction energies $\omega_{\gamma, i}(L)$ and the line interaction energy $\omega_{\tau}(L)$. Please note that in the limit of infinitely large separation between the walls these interaction contributions vanish: $\omega_{\gamma, i}(L \rightarrow \infty) \rightarrow 0$ and $\omega_{\tau}(L \rightarrow \infty) \rightarrow 0$.

The ultimate goal here is to infer properties of the total effective interaction between two colloidal particles in Fig. 1. (a). As the total volume of medium $i \in\{1,2\}$ (see the space outside of the colloids in Fig. 1(a)) and the total area of the fluidfluid interface (see the green line outside of the colloids in Fig. 1(a)) do not change as functions of the distance between the colloids, i.e., of $L$. Hence the terms involving $\Omega_{b, i}$ and $\gamma_{1,2}$ in Eq. (6), which quantifies only the contributions from inside the dashed box in Fig.11a), are compensated by contributions from outside the box when one considers the effective interaction of the total system and can be disregarded in the following. Therefore, the total effective inter-surface interaction is solely determined by the two quantities $\omega_{\gamma, i}(L)$ and $\omega_{\tau}(L)$.

\section{RESULTS AND DISCUSSION}

\section{A. Electrostatic potential}

\section{Exact calculation}

The exact electrostatic potential $\Psi_{i}^{e}(x, z)$ everywhere inside our system must satisfy the DH equation (Eq. (4)) along with the boundary conditions listed below Eq. (4). In order to achieve such a solution, we first split the actual problem depicted in Fig. 1b) into three sub-problems: (i) two walls situated at $z=0$ and $z=L$ with charge densities $\sigma_{1}^{0}$ and $\sigma_{1}^{L}$, respectively, and the space in between the walls is filled with medium "1" only, (ii) the same set-up as in part (i) but with the fluid medium " 2 " instead of " 1 " and consequently, the charge densities at the walls replaced by $\sigma_{2}^{0}$ and $\sigma_{2}^{L}$, and (iii) two fluids separated by an interface at $x=0$ in the absence of any 
walls. For all these sub-problems, the electrostatic potential is obtained by solving the $\mathrm{DH}$ equation. Since this is a linear equation, we, by fiat, add the solution of sub-problem (i) with the solution obtained for the lower half space $(x<0)$ in subproblem (iii) and the solution of sub-problem (ii) with the one obtained for the upper half-space $(x>0)$ in sub-problem (iii). It turns out that the resulting solutions after performing such additions satisfy all the required boundary conditions except for the continuity of the electrostatic potential at the interface.
This discrepancy is eliminated by adding a correction function which is also solution of the DH equation and which takes care of the continuity problem at the interface while keeping the already satisfied boundary conditions unaltered. Construction of a function like this is accomplished by means of Fourier series expansion (for details, please refer to Chapter III of Ref. [37]). Finally, putting everything together, one arrives at the following final expressions for the electrostatic potential in the two media:

$$
\begin{aligned}
\Psi_{i}^{e}(x, z)= & \frac{\sigma_{i}^{0} \cosh \left(\kappa_{i}(L-z)\right)+\sigma_{i}^{L} \cosh \left(\kappa_{i} z\right)}{\varepsilon_{i} \kappa_{i} \sinh \left(\kappa_{i} L\right)}+\Psi_{b, i}+\sum_{j \in\{1,2\}}^{j \neq i}\left[\frac{(-1)^{j} \kappa_{j} \varepsilon_{j} \Psi_{D}}{\kappa_{1} \varepsilon_{1}+\kappa_{2} \varepsilon_{2}} e^{-\kappa_{i}|x|}+\frac{1}{\kappa_{1} \varepsilon_{1}+\kappa_{2} \varepsilon_{2}}\left(\frac{\sigma_{j}^{0}+\sigma_{j}^{L}}{\kappa_{j}}-\frac{\kappa_{j} \varepsilon_{j}}{\kappa_{i} \varepsilon_{i}} \frac{\sigma_{i}^{0}+\sigma_{i}^{L}}{\kappa_{i}}\right) \frac{e^{-\kappa_{i}|x|}}{L}\right. \\
& \left.+\sum_{n=1}^{\infty} \frac{2}{p_{1}^{(n)} \varepsilon_{1}+p_{2}^{(n)} \varepsilon_{2}}\left(\frac{\sigma_{j}^{0}+(-1)^{n} \sigma_{j}^{L}}{p_{j}^{(n)}}-\frac{p_{j}^{(n)} \varepsilon_{j}}{p_{i}^{(n)} \varepsilon_{i}} \frac{\sigma_{i}^{0}+(-1)^{n} \sigma_{i}^{L}}{p_{i}^{(n)}}\right) \frac{e^{-p_{i}^{(n)}|x|}}{L} \cos \left(\frac{n \pi z}{L}\right)\right]
\end{aligned}
$$

with $p_{m}^{(n)}=\sqrt{\left(\frac{n \pi}{L}\right)^{2}+\kappa_{m}^{2}}$. The first term of this expression represents the solution of the sub-problem (i) or (ii) depending upon the medium where the potential is looked at, the second term and the first term of the sum over $j$ together is the solution of sub-problem (iii) and the rest is the correction function. Some of the limiting cases and boundary conditions can be verified easily from Eq. (7). For example, in the limit $x \rightarrow \pm \infty$, all the terms which vary exponentially with $x$ vanish and one is left with the first two terms which are the solutions inside the two media in the presence of the walls but far away from the fluid-fluid interface at $x=0$. In the limit $L \rightarrow \infty$ with $\sigma_{1}^{L}=\sigma_{2}^{L}=0$, the first term of Eq. (7) simplifies to an exponentially decaying potential of a single charged wall, the summation term $\propto 1 / L$ in the first line vanishes, and the sum over $n$ in the second line can be converted to an integral over $q=n \pi / L$; see Eq. (8) below. Therefore, Eq. (7) reduces to the potential for a single charged wall placed at $z=0$ in contact with two electrolytes spanning the region $z>0$. For both $x, L \rightarrow \infty$, only the first two terms do not vanish with the first term reduced to an exponentially decaying function characteristic of a single charged wall in contact with an electrolyte. Finally, again from Eq. (7), at $z=0$ and $z=L$, one easily verifies the relations $\varepsilon_{i} \partial_{z} \Psi_{i}^{e}(z=0)=-\sigma_{i}^{0}$ and $\varepsilon_{i} \partial_{z} \Psi_{i}^{e}(z=L)=\sigma_{i}^{L}$ since the $z$-derivative of all the terms except the first one vanish. When all the charge densities are set to zero, only the second and the third terms in Eq. (7) do not vanish which forms the solution of the sub-problem (iii) and, on top of that, if one approaches $x \rightarrow \pm \infty, \Psi_{i}^{e}$ reduces to $\Psi_{b, i}$ which is the potential in the bulk of two media far away from the interface.

\section{Superposition approximation}

Since DH equation is linear, a superposition approximation, in principle, can be used to obtain the potential distribution for a two-body problem. This requires one to first calculate the potential due to each object in the absence of the other and then to simply add these two potentials at each point in space to obtain an approximate potential in the presence of both particles. Accordingly, we first calculate the electrostatic potential due to a single charged wall placed at $z=0$ and carrying charge densities $\sigma_{i}^{0}$ in contact with medium $i \in\{1,2\}$ filling the space $z>0$ with the fluid-fluid interface formed at $x=0$. Following the same procedure as described above, we again divide the problem into the following sub-problems: (i) a single wall placed at $z=0$ in contact with medium " 1 " and carrying charge density $\sigma_{1}^{0}$, (ii) a single wall place at $z=0$ with medium " 2 " and carrying charge density $\sigma_{2}^{0}$, (iii) two fluid media separated by an interface at $x=0$ in the absence of any wall. By adding the solutions of these problems in the respective medium, one obtains a solution which satisfies all the required boundary conditions except the continuity of the potential at the interface. This is rectified by constructing again a correction function in the same fashion as before albeit by means of Fourier transforms. Finally, the electrostatic potential due to a single charged wall at $z=0$ is given by

$$
\begin{aligned}
& \Psi_{i}^{\sin }(x, z)=\frac{\sigma_{i}^{0}}{\varepsilon_{i} \kappa_{i}} e^{-\kappa_{i} z}+\Psi_{b, i}+\sum_{j \in\{1,2\}}^{j \neq i}\left[\frac{(-1)^{j} \kappa_{j} \varepsilon_{j} \Psi_{D}}{\kappa_{1} \varepsilon_{1}+\kappa_{2} \varepsilon_{2}} e^{-\kappa_{i}|x|}\right. \\
& \left.+\frac{1}{\pi} \int_{-\infty}^{\infty} d q \frac{e^{-p_{i}(q)|x|} \cos (q z)}{p_{1}(q) \varepsilon_{1}+p_{2}(q) \varepsilon_{2}}\left(\frac{\sigma_{j}^{0}}{p_{j}(q)}-\frac{p_{j}(q) \varepsilon_{j}}{p_{i}(q) \varepsilon_{i}} \frac{\sigma_{i}^{0}}{p_{i}(q)}\right)\right],
\end{aligned}
$$

with $p_{m}(q)=\sqrt{q^{2}+\kappa_{m}^{2}}$. From this expression, one readily verifies the relation $\varepsilon_{i} \partial_{z} \Psi_{i}^{\sin }(z=0)=-\sigma_{i}^{0}$. In the limit $x \rightarrow \pm \infty$ all the terms that vary exponentially with $x$ vanish and one is left with the first two terms in Eq. (8) which is nothing but the usual screened potential due to a single charged wall plus the potential $\Psi_{b, i}$ due to partitioning of ions at the interface. Setting all the charge densities to zero, one recovers the potential distribution due to the presence of a fluid interface in the absence of any wall and on top of that, in the limit $x \rightarrow \pm \infty$, 
Eq. (8) reduces to the bulk potential $\Psi_{b, i}$.

Next, a similar solution is obtained for a wall placed at $z=$ $L$ carrying charge densities $\sigma_{1}^{L}$ and $\sigma_{2}^{L}$ in the absence of any wall at $z=0$. It can be obtained readily from Eq. (8) without an explicit calculation by just replacing $z$ and $\sigma_{i}^{0}$ with $L-z$ and
$\sigma_{i}^{L}$, respectively. Then by adding these two solutions for walls present at $z=0$ and $z=L$ in the absence of each other, one obtains the following expression for the electrostatic potential $\Psi_{i}^{s}(x, z)$ in the presence of both walls under the superposition approximation:

$$
\begin{aligned}
\Psi_{i}^{s}(x, z) & =\frac{\sigma_{i}^{0} e^{-\kappa_{i} z}+\sigma_{i}^{L} e^{\kappa_{i}(z-L)}}{\kappa_{i} \varepsilon_{i}}+2 \Psi_{b, i}+\sum_{j \in\{1,2\}}^{j \neq i} \frac{(-1)^{j} 2 \kappa_{j} \varepsilon_{j} \Psi_{D}}{\kappa_{1} \varepsilon_{1}+\kappa_{2} \varepsilon_{2}} e^{-\kappa_{i}|x|} \\
& +\sum_{j \in\{1,2\}}^{j \neq i} \frac{1}{\pi} \int_{-\infty}^{\infty} d q \frac{(-1)^{j} e^{-p_{i}(q)|x|}}{p_{1}(q) \varepsilon_{1}+p_{2}(q) \varepsilon_{2}}\left\{\left(\frac{\sigma_{j}^{0}}{p_{j}(q)}-\frac{p_{j}(q) \varepsilon_{j}}{p_{i}(q) \varepsilon_{i}} \frac{\sigma_{i}^{0}}{p_{i}(q)}\right) \cos (q z)+\left(\frac{\sigma_{j}^{L}}{p_{j}(q)}-\frac{p_{j}(q) \varepsilon_{j}}{p_{i}(q) \varepsilon_{i}} \frac{\sigma_{i}^{L}}{p_{i}(q)}\right) \cos (-q(z-L))\right\} .
\end{aligned}
$$

It is worth noting that $\Psi_{i}^{s}(x, z)$ fails to behave properly in most of the limiting cases and to satisfy the boundary conditions listed below Eq. (4). At the two boundaries, $\varepsilon_{i} \partial_{z} \Psi_{i}^{s}(z=0) \neq$ $-\sigma_{i}^{0}$ and $\varepsilon_{i} \partial_{z} \Psi_{i}^{S}(z=L) \neq \sigma_{i}^{L}$. In the limit $L \rightarrow \infty$ with $\sigma_{1}^{L}=\sigma_{2}^{L}=0, \Psi_{i}^{s}(x, z)$ does not reduce to the expression for the potential due to a single wall at $z=0$ given in Eq. [8]. Setting all the charge densities to zero and $x \rightarrow \pm \infty$, one does not even recover the bulk potential $\Psi_{b, i}$. We like to finish our discussion on the electrostatic potential by pointing out that for the case of identical particles, i.e., for $\sigma_{i}^{0}=\sigma_{i}^{L}$, Eqs. (7) and (9) reduce to Eq. (1) and (2) of Ref. [20], respectively. Please note that the walls in Ref. [20] are situated at $z= \pm L$ whereas in the current set-up they are situated at $z=0$ and $L$ which needs to be taken into account for proper transformations. Plots showing comparisons of the two potential distributions (Eqs. (7) and (9)) are displayed in Chapter III of Ref. [37].

\section{B. Interaction parameters}

Once the electrostatic potential is known, it can be inserted back into the grand potential functional to obtain $\widetilde{\Omega}(L)=$ $\widetilde{\Omega}\left[n_{ \pm}[\Psi]\right]$ and all the interaction parameters described in Eq. (6) can be extracted. This is done by identifying the terms proportional to $V_{i}, A_{i}, A_{1,2}$ and $\ell$, and separating the $L$-independent parts from the $L$-dependent parts in the expression for $\widetilde{\Omega}(L)$. Calculating interaction parameters within the superposition approximation implies using expression for the electrostatic potential given in Eq. (9) but no further superposition is performed for any other quantities.

\section{Surface interaction energy}

Surface interaction energy density $\omega_{\gamma, i}(L)$, as defined in Eq. (6), is given within the exact calculation by

$$
\omega_{\gamma, i}^{e}(L)=\left(\left(\sigma_{i}^{0}\right)^{2}+\left(\sigma_{i}^{L}\right)^{2}\right) \frac{\operatorname{coth}\left(\kappa_{i} L\right)-1}{4 \kappa_{i} \varepsilon_{i}}+\frac{\sigma_{i}^{0} \sigma_{i}^{L}}{2 \kappa_{i} \varepsilon_{i} \sinh \left(\kappa_{i} L\right)} .
$$

For $\sigma_{i}^{0}=\sigma_{i}^{L}=0$, there is no electrostatic interaction between the surfaces, i.e., $\omega_{\gamma, i}^{e}(L)=0$ for any separation $L$ between the walls. In case of $\sigma_{i}^{0}=-\sigma_{i}^{L}$, using L'Hôpital's rule, one obtains from Eq. (10) a finite, i.e., non-divergent, attractive surface interaction in the limit of vanishing separations: $\omega_{\gamma, i}^{e}(L \rightarrow 0)=-\frac{\left(\sigma_{i}^{0}\right)^{2}}{2 \kappa_{i} \varepsilon_{i}}$. In contrast, for $\sigma_{i}^{0} \neq-\sigma_{i}^{L}$, both $\operatorname{coth}\left(\kappa_{i} L\right)$ and $\frac{1}{\sinh \left(\kappa_{i} L\right)}$ diverge $\sim \frac{1}{L}$, and, as a result, $\omega_{\gamma, i}^{e}$ diverges to $+\infty$ in the limit $L \rightarrow 0$, i.e., $\omega_{\gamma, i}^{e}(L \rightarrow 0)$ becomes repulsive. In the opposite limit, i.e., for $L \rightarrow \infty$, one also has two different cases depending upon the charge densities. For $\sigma_{i}^{0} \sigma_{i}^{L} \neq 0, \omega_{\gamma, i}^{e}(L \rightarrow \infty) \simeq \frac{1}{\kappa_{i} \varepsilon_{i}}\left[\frac{\left(\sigma_{i}^{0}\right)^{2}+\left(\sigma_{i}^{L}\right)^{2}}{2} e^{-2 \kappa_{i} L}+\sigma_{i}^{0} \sigma_{i}^{L} e^{-\kappa_{i} L}\right]$, i.e., to the leading order $\omega_{\gamma, i}^{e}$ varies $\sim e^{-\kappa_{i} L}$. However, if one of the two charge densities $\sigma_{i}^{0}$ and $\sigma_{i}^{0}$ vanishes, the decay becomes twice as slow $\left(\sim e^{-2 \kappa_{i} L}\right)$. The latter effect is related to the finite size of the system. The overall decay of $\omega_{\gamma, i}^{e}(L)$ is monotonic unless the conditions $\sigma_{i}^{0} \sigma_{i}^{L}<0$ and $\sigma_{i}^{0} \neq-\sigma_{i}^{L}$ are simultaneously satisfied. In this case, $\omega_{\gamma, i}^{e}(L \rightarrow 0) \rightarrow+\infty$ and one can verify easily from Eq. (10) that $\omega_{\gamma, i}^{e}(L)$ has a zero at $L_{0}=-\frac{1}{\kappa_{i}} \ln \left(\frac{-2 \sigma_{i}^{0} \sigma_{i}^{L}}{\left(\sigma_{i}^{0}\right)^{2}+\left(\sigma_{i}^{L}\right)^{2}}\right)$, and an extremum at $L_{\mathrm{ex}}=$ $\frac{1}{\kappa_{i}} \operatorname{arcosh}\left(-\frac{1}{2}\left(\frac{\sigma_{i}^{0}}{\sigma_{i}^{L}}+\frac{\sigma_{i}^{L}}{\sigma_{i}^{0}}\right)\right)$. Since $\omega_{\gamma, i}^{e}(L \rightarrow 0) \rightarrow+\infty$, this extremum must be a minimum. Therefore, with increasing separation $L$, the surface interaction energy density is initially positive, then becomes negative, shows a minimum and eventually vanishes for large separations as $\sim e^{-\kappa_{i} L}$.

Within the superposition approximation, the surface inter- 

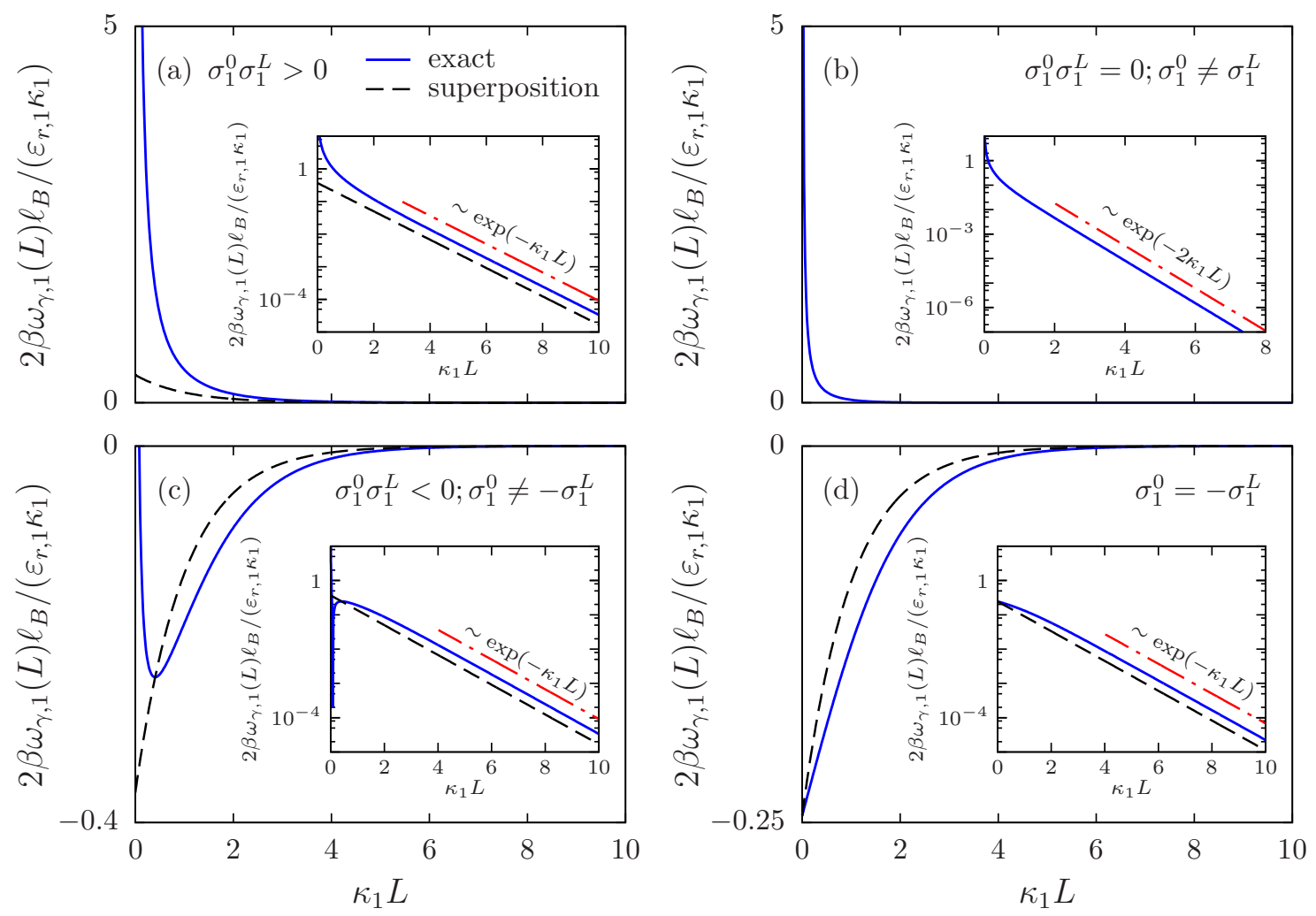

FIG. 2. Variation of the surface interaction energy densities $\omega_{\gamma, 1}^{e}(L)$ and $\omega_{\gamma, 1}^{s}(L)$, both expressed in units of $\varepsilon_{r, 1} \kappa_{1} /\left(2 \beta \ell_{B}\right)$, as functions of the scaled separation $\kappa_{1} L$ between the walls for (a) $\sigma_{1}^{0} \sigma_{1}^{L}>0$, (b) $\sigma_{1}^{0} \sigma_{1}^{L}=0$ with $\sigma_{1}^{0} \neq \sigma_{1}^{L}$, (c) $\sigma_{1}^{0} \sigma_{1}^{L}<0$ with $\sigma_{1}^{0} \neq-\sigma_{1}^{L}$, and (d) $\sigma_{1}^{0}=-\sigma_{1}^{L}$. As shown by the plots, $\omega_{\gamma, 1}^{e}(L)$ diverges in the limit of vanishing separation between the walls except for the case considered in panel (d), whereas $\omega_{\gamma, 1}^{s}(L)$ is always finite in this limit. For the special case of $\sigma_{1}^{0}=-\sigma_{1}^{L}$ considered in panel (d), both $\omega_{\gamma, 1}^{e}(0)$ and $\omega_{\gamma, 1}^{s}(0)$ are finite and $\omega_{\gamma, 1}^{e}(0)=\omega_{\gamma, 1}^{s}(0)$. In the opposite limit, i.e., in the limit of infinitely large separations between the walls, both $\omega_{\gamma, 1}^{e}$ and $\omega_{\gamma, 1}^{s}$, if non-zero, decay exponentially which in each case is confirmed by the semi-logarithmic plots in the insets. However, when one of the two charge densities are zero (panel (b)), $\omega_{\gamma, 1}^{s}(L)$ is zero for any separation between the walls whereas $\omega_{\gamma, 1}^{e}(L)$ is non-zero but decays twice as slow compared to the other cases. The overall decay of the surface interaction energies are monotonic except when the walls are oppositely charged with $\sigma_{1}^{0} \neq-\sigma_{1}^{L}$ In this case, the exact surface interaction energy shows a minimum before decaying to zero at large separations.

action energy density is given by

$$
\omega_{\gamma, i}^{s}(L)=\frac{\sigma_{i}^{0} \sigma_{i}^{L}}{2 \kappa_{i} \varepsilon_{i}} e^{-\kappa_{i} L} .
$$

In the special case of $\sigma_{i}^{0}=-\sigma_{i}^{L}, \omega_{\gamma, i}^{s}(0)=\omega_{\gamma, i}^{e}(0)$. However, for $\sigma_{i}^{0} \sigma_{i}^{L}=0$, there is no surface interaction within the superposition approximation, i.e., $\omega_{\gamma, i}^{s}(L)=0$ for any given separation between the walls which is not the case for the exact solution where one has a non-zero surface interaction even if one of the two surface charge densities vanishes. Moreover, for $\sigma_{i}^{0} \sigma_{i}^{L} \neq 0$, contrary to the results obtained within the exact calculation, $\omega_{\gamma, i}^{s}(L)$ is always (i) finite in the limit of vanishing separation between the walls, and (ii) decays monotonically with the separation length $L$. Although Eq. (11) predicts the exponential decay at large separations correctly, the prefactor is too small by a factor of 2 compared to the exact result, i.e., $\omega_{\gamma, i}^{e}(L \rightarrow \infty) / \omega_{\gamma, i}^{s}(L \rightarrow \infty)=2$. Please note that a similar factor of 2 is present for the case of identically charged walls as well [20] which, as is confirmed by the present analysis, cannot be related to a symmetry of the surface charge distribution.

The surface interaction energy densities (expressed in units of $\left.\varepsilon_{r, 1} \kappa_{1} /\left(2 \beta \ell_{B}\right)\right)$ in medium "1" within the exact (Eq. 100) and the superposition (Eq. (11)) approach as functions of the scaled separation $\kappa_{1} L$ between the walls are compared in Fig. 2 for different combinations of the charge densities $\sigma_{1}^{0}$ and $\sigma_{1}^{L}$ at the walls. Please note that for typical system parameters the scale-factor $\varepsilon_{r, 1} \kappa_{1} /\left(2 \beta \ell_{B}\right)$ in aqueous electrolyte solution with $\kappa_{1}=0.1 \mathrm{~nm}^{-1}$ is $\approx 0.3 \mathrm{mN} / \mathrm{m}$. Although not shown in the plots, $\omega_{\gamma, 2}^{e}(L)$ and $\omega_{\gamma, 2}^{s}(L)$ behave similarly to $\omega_{\gamma, 1}^{e}(L)$ and $\omega_{\gamma, 1}^{s}(L)$, respectively.

\section{Line interaction energy}

The line interaction energy density $\omega_{\tau}(L)$, as defined in Eq. (6), is given within the exact calculation by 
TABLE I. Values used for the plots in Fig. 3 of the charge densities $\sigma_{1}^{0}, \sigma_{1}^{L}, \sigma_{2}^{0}$, and $\sigma_{2}^{L}$ in units of $e /$ nm $^{2}$, inverse Debye lengths $\kappa_{1}$ and $\kappa_{2}$ in medium " 1 " and " 2 ", respectively in units of $\mathrm{nm}^{-1}$, relative permittivities $\varepsilon_{r, 1}$ and $\varepsilon_{r, 2}$ of medium "1" and " 2 ", respectively, the Donnan potential $\Psi_{D}$ in units of $1 /(\beta e)$ and the Bjerrum length $\ell_{B}$ in units of nm.

\begin{tabular}{|c|c|c|c|c|c|c|c|c|c|c|}
\hline$\overline{\text { Figure } 3}$ & $\overline{\sigma_{1}^{0}\left(e / \mathrm{nm}^{2}\right)}$ & $\sigma_{1}^{L}\left(e / \mathrm{nm}^{2}\right)$ & $\sigma_{2}^{0}\left(e / \mathrm{nm}^{2}\right)$ & $\sigma_{2}^{L}\left(e / \mathrm{nm}^{2}\right)$ & $\kappa_{1}\left(\mathrm{~nm}^{-1}\right)$ & $\overline{\kappa_{2}}\left(\mathrm{~nm}^{-1}\right)$ & $\varepsilon_{r, 1}$ & $\varepsilon_{r, 2}$ & $\beta e \Psi_{D}$ & $\ell_{B}(\mathrm{~nm})$ \\
\hline (a) & 0.02 & 0.03 & -0.0004 & 0.0002 & 0.1 & 0.03 & 80 & 2 & 1 & 55.7 \\
\hline (b) & -0.02 & -0.03 & 0.0004 & -0.0002 & 0.1 & 0.03 & 80 & 2 & 1 & 55.7 \\
\hline (c) & 0.02 & -0.03 & 0.0004 & 0.0002 & 0.1 & 0.03 & 80 & 2 & 1 & 55.7 \\
\hline (d) & -0.02 & 0.03 & -0.0004 & -0.0002 & 0.1 & 0.03 & 80 & 2 & 1 & 55.7 \\
\hline (e) & 0.02 & -0.02 & 0.0002 & -0.0002 & 0.1 & 0.03 & 80 & 2 & 1 & 55.7 \\
\hline (f) & 0.02 & 0 & 0.0004 & 0 & 0.1 & 0.03 & 80 & 2 & 1 & 55.7 \\
\hline (g) & 0.02 & 0 & 0 & 0.0002 & 0.1 & 0.03 & 80 & 2 & 1 & 55.7 \\
\hline (h) & 0.02 & 0.03 & 0 & 0.0002 & 0.1 & 0.03 & 80 & 2 & 1 & 55.7 \\
\hline
\end{tabular}

$$
\begin{aligned}
\omega_{\tau}^{e}(L) & =\frac{1}{L} \frac{1}{\kappa_{1} \varepsilon_{1}+\kappa_{2} \varepsilon_{2}}\left(\frac{\left(\sigma_{1}^{0}+\sigma_{1}^{L}\right)\left(\sigma_{2}^{0}+\sigma_{2}^{L}\right)}{2 \kappa_{1} \kappa_{2}}-\frac{\kappa_{2} \varepsilon_{2}}{\kappa_{1} \varepsilon_{1}}\left(\frac{\sigma_{1}^{0}+\sigma_{1}^{L}}{2 \kappa_{1}}\right)^{2}-\frac{\kappa_{1} \varepsilon_{1}}{\kappa_{2} \varepsilon_{2}}\left(\frac{\sigma_{2}^{0}+\sigma_{2}^{L}}{2 \kappa_{2}}\right)^{2}\right) \\
& +\frac{1}{2 L} \sum_{n=1}^{\infty} \frac{1}{p_{1}^{(n)} \varepsilon_{1}+p_{2}^{(n)} \varepsilon_{2}}\left(\frac{2\left(\sigma_{1}^{0}+(-1)^{n} \sigma_{1}^{L}\right)\left(\sigma_{2}^{0}+(-1)^{n} \sigma_{2}^{L}\right)}{p_{1}^{(n)} p_{2}^{(n)}}-\frac{p_{2}^{(n)} \varepsilon_{2}}{p_{1}^{(n)} \varepsilon_{1}}\left(\frac{\sigma_{1}^{0}+(-1)^{n} \sigma_{1}^{L}}{p_{1}^{(n)}}\right)^{2}-\frac{p_{1}^{(n)} \varepsilon_{1}}{p_{2}^{(n)} \varepsilon_{2}}\left(\frac{\sigma_{2}^{0}+(-1)^{n} \sigma_{2}^{L}}{p_{2}^{(n)}}\right)^{2}\right) \\
& -\frac{1}{2 \pi} \int_{0}^{\infty} \frac{d q}{p_{1}(q) \varepsilon_{1}+p_{2}(q) \varepsilon_{2}}\left(\frac{2\left(\sigma_{1}^{0} \sigma_{2}^{0}+\sigma_{1}^{L} \sigma_{2}^{L}\right)}{p_{1}(q) p_{2}(q)}-\frac{p_{2}(q) \varepsilon_{2}}{p_{1}(q) \varepsilon_{1}}\left(\frac{\left(\sigma_{1}^{0}\right)^{2}+\left(\sigma_{1}^{L}\right)^{2}}{p_{1}(q)^{2}}\right)-\frac{p_{1}(q) \varepsilon_{1}}{p_{2}(q) \varepsilon_{2}}\left(\frac{\left(\sigma_{2}^{0}\right)^{2}+\left(\sigma_{2}^{L}\right)^{2}}{p_{2}(q)^{2}}\right)\right),
\end{aligned}
$$

and within the superposition approximation by

$$
\begin{aligned}
\omega_{\tau}^{s}(L) & =\frac{\kappa_{1} \varepsilon_{1} \Psi_{D}}{4\left(\kappa_{1} \varepsilon_{1}+\kappa_{2} \varepsilon_{2}\right)}\left(\frac{\sigma_{2}^{0}+\sigma_{2}^{L}}{\kappa_{2}}-\frac{\kappa_{2} \varepsilon_{2}}{\kappa_{1} \varepsilon_{1}} \frac{\sigma_{1}^{0}+\sigma_{1}^{L}}{\kappa_{1}}\right)-\frac{\Psi_{D}}{4 \pi} \int_{-\infty}^{\infty} \frac{p_{1}(q) \varepsilon_{1}}{p_{1}(q) \varepsilon_{1}+p_{2}(q) \varepsilon_{2}}\left(\frac{\sigma_{2}^{0}+\sigma_{2}^{L}}{p_{2}(q)}-\frac{p_{2}(q) \varepsilon_{2}}{p_{1}(q) \varepsilon_{1}} \frac{\sigma_{1}^{0}+\sigma_{1}^{L}}{p_{1}(q)}\right) \frac{\sin (q L)}{q} d q \\
& +\frac{1}{2 \pi} \int_{-\infty}^{\infty} \frac{\cos (q L) d q}{p_{1}(q) \varepsilon_{1}+p_{2}(q) \varepsilon_{2}}\left(\frac{\sigma_{1}^{0} \sigma_{2}^{L}+\sigma_{2}^{0} \sigma_{1}^{L}}{p_{1}(q) p_{2}(q)}-\frac{p_{2}(q) \varepsilon_{2}}{p_{1}(q) \varepsilon_{1}} \frac{\sigma_{1}^{0} \sigma_{1}^{L}}{p_{1}(q)^{2}}-\frac{p_{1}(q) \varepsilon_{1}}{p_{2}(q) \varepsilon_{2}} \frac{\sigma_{2}^{0} \sigma_{2}^{L}}{p_{2}(q)^{2}}\right),
\end{aligned}
$$

with $p_{m}^{(n)}$ and $p_{m}(q)$ as defined earlier after Eq. (7) and Eq. (8), respectively. In general, in the limit of vanishing separation between the walls, $\omega_{\tau}^{e}$ in Eq. (12) diverges $\sim \frac{1}{L}$. On the other hand, in the limit $L \rightarrow 0$, the second term of Eq. (13) vanish whereas the other terms have a finite non-zero value. Consequently, $\omega_{\tau}^{s}$ also remains finite in this limit. In the opposite limit, i.e., for $L \rightarrow \infty$, the sum in the second line of Eq. (12) is cancelled by the integral in the third line. The first term also vanishes in this limit. However, the difference of the sum and the integral in Eq. (12) such that $\omega_{\tau}^{e}$ decays exponentially to zero in the limit of large separation between the walls. The superposition expression (13) also decays exponentially to zero in the limit of large separation. As it is difficult to analyze the overall variation of the line interactions analytically, we have plotted it in Fig. 3 for different combination of the four charge densities $\sigma_{1}^{0}, \sigma_{1}^{L}, \sigma_{2}^{0}$, and $\sigma_{2}^{L}$, which are specified in Table \] As one can see from the plots, unless the two walls are $e x$ actly oppositely charged (as it is the case in Fig. 3( e)), the line interaction within the exact calculation diverges whereas it remains finite for the superposition approximation in the limit $L \rightarrow 0$. For exactly oppositely charged walls, both $\omega_{\tau}^{e}$ and $\omega_{\tau}^{s}$ remain finite and have the same value at $L=0$ (see Fig. 3(e)). If all four charge densities are non-zero (cases considered in Figs. [3 (a)-3 (e); see Table @), the exponential decay at large separations is characterized by $\sim e^{-\kappa_{2} L}$ since we have assumed $\kappa_{2}<\kappa_{1}$ (see Table【). The decay is shown only in Fig. 3 (e) for the sake of neatness. However, even when both $\omega_{\tau}^{e}$ and $\omega_{\tau}^{s}$ decay as $\sim e^{-\kappa_{2} L}$, the latter is too small compared to the former by a factor of 2 (see for example, the inset of Fig. 3 (e)). If one of the walls is uncharged, $\omega_{\tau}^{s}$ still varies as $\sim e^{-\kappa_{2} L}$ but 

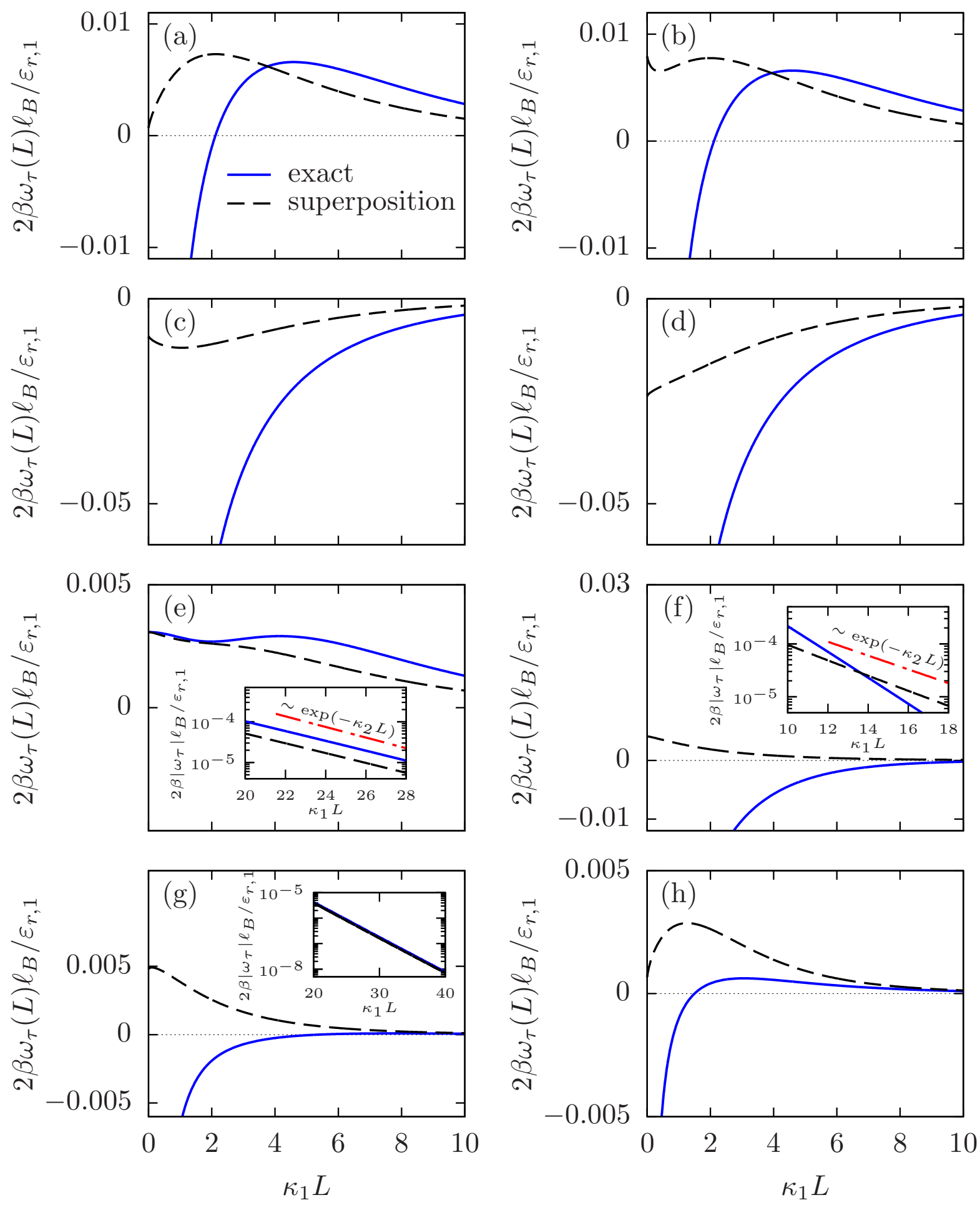

FIG. 3. Line interaction energy $\omega_{\tau}(L)$ within both the exact and the superposition calculations expressed in units of $\varepsilon_{r, 1} /\left(2 \beta \ell_{B}\right)$ as functions of the scaled separation length $\kappa_{1} L$ for different combinations of the charge densities $\sigma_{1}^{0}, \sigma_{1}^{L}, \sigma_{2}^{0}$, and $\sigma_{2}^{L}$ specified in Table $\square$ As it is clear from the plots, both $\omega_{\tau}^{e}$ and $\omega_{\tau}^{s}$ can vary monotonically as well as non-monotonically depending upon the parameters and in most cases the superposition approximation fails to capture the correct behavior properly. In the limit of vanishing separation between the walls, $\omega_{\tau}^{s}$ stays finite, whereas $\omega_{\tau}^{e}$ usually diverges unless the two walls are exactly oppositely charged (panel (e)). The decay at large separations is always exponential (shown, for example, in the inset of panel (e) but the decay rate for $\omega_{\tau}^{e}$ and $\omega_{\tau}^{s}$ differs by a factor of 2 when one of the walls is uncharged as shown by the semi-logarithmic plot in the inset of panel (f)). Moreover, even when the superposition expression predicts the correct decay rate it always underestimates the magnitude by a factor of 2 if none of the four charge densities are zero. This can be seen from the corresponding curves in the inset of panel (e), which, instead of falling on top of each other, are parallel to each other. However, when either one of the four or two diagonally opposite charge densities are zero, the ratio $\omega_{\tau}^{e}(L \rightarrow \infty) / \omega_{\tau}^{s}(L \rightarrow \infty)$ converges to a value which depends upon the system parameters. For the parameters used in panel $(\mathrm{g})$ and $(\mathrm{h})$, it is close to unity. 

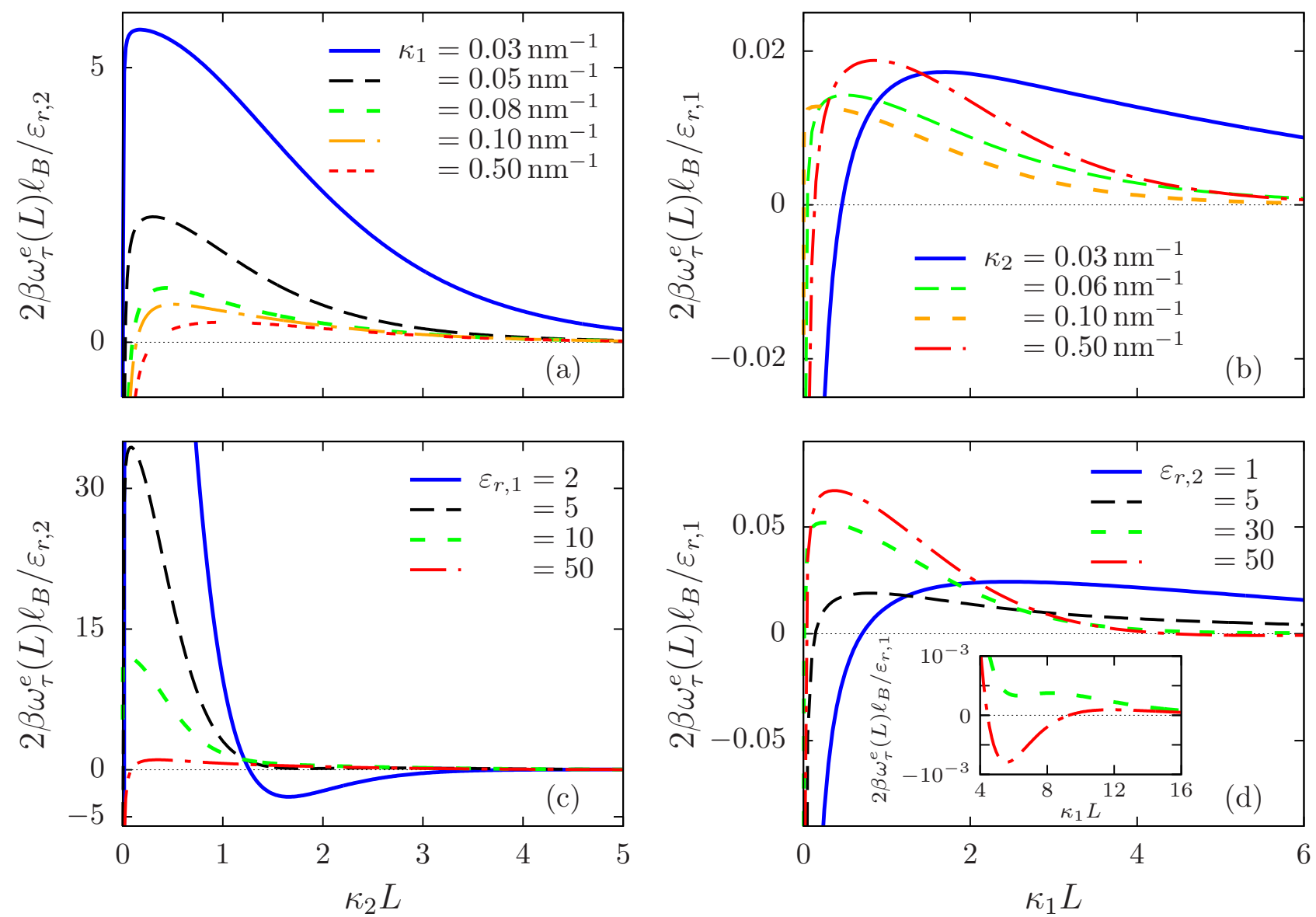

FIG. 4. Variation of the exact line interaction energy density $\omega_{\tau}^{e}(L)$ (expressed in units of $\varepsilon_{r, 2} /\left(2 \beta \ell_{B}\right)$ in the left panels and $\varepsilon_{r, 1} /\left(2 \beta \ell_{B}\right)$ in the right panels) as function of the scaled separation $\kappa_{2} L$ (left panels) and $\kappa_{1} L$ (right panels) for varying (a) inverse Debye length $\kappa_{1}$ in medium "1", (b) inverse Debye length $\kappa_{2}$ in medium " 2 ", (c) relative permittivity $\varepsilon_{r, 1}$ of medium "1", and (d) relative permittivity $\varepsilon_{r, 2}$ of medium " 2 ". The other parameters used for the plots are: $\sigma_{1}^{0}=0.02 \mathrm{e} / \mathrm{nm}^{2}, \sigma_{1}^{L}=-0.03 \mathrm{e} / \mathrm{nm}^{2}, \sigma_{2}^{0}=-0.0004 \mathrm{e} / \mathrm{nm}^{2}, \sigma_{2}^{L}=0.0002 \mathrm{e} / \mathrm{nm}^{2}$, and $\beta e \Psi_{D}=1$. Unless otherwise stated, $\kappa_{1}=0.1 \mathrm{~nm}^{-1}, \kappa_{2}=0.03 \mathrm{~nm}^{-1}, \varepsilon_{r, 1}=80$, and $\varepsilon_{r, 2}=2$ are considered. As one can see, $\omega_{\tau}^{e}(L)$ always show at least one maximum. However, with decreasing $\varepsilon_{r, 1}$, a single minimum can occur while with increasing $\varepsilon_{r, 2}$, a minimum and a second maximum can occur.

$\omega_{\tau}^{e}$ decays as $\sim e^{-2 \kappa_{2} L}$ (see Fig. 3(f)). Not only that, if two diagonally opposite charge densities out of the four are zero or if only one of the four charge densities is zero (cases considered in Figs. $3(\mathrm{~g})$ and $3 \mathrm{~h}$ ); see Table I), or if two of the four charge densities, which are facing each other, are zero, then the ratio $\omega_{\tau}^{e}(L \rightarrow \infty) / \omega_{\tau}^{s}(L \rightarrow \infty)$ converges to a value which is not generally fixed but which depends upon the parameters of the system, especially the Donnan potential $\Psi_{D}$ (for details, see section 16.3.10 of Ref. [37]). As is clear from Fig. 3, the overall variation of both, $\omega_{\tau}^{e}(L)$ and $\omega_{\tau}^{s}(L)$, with the separation distance $L$ can be non-monotonic but in most cases the superposition result cannot capture the correct variation. Another important observation is that the exact expression for the line interaction energy density (Eq. (12)) is insensitive to changes in the signs of the charge densities when the sign of all four of them are reversed. Clearly, the superposition expression (Eq. (13) ) lacks this property in general as Eq. (13) contains $\sigma$-terms which are odd as well as $\sigma$-terms which are even. This particular feature can also be verified by comparing
Figs. 3 (a) and 3 b) or Figs. 3 (c) and 3(d). We have checked the other combinations of the four charge densities as well, but concluded that the behavior can be broadly classified into the eight different cases presented in Fig. 3

Figure 4 shows the variation of the line interaction $\omega_{\tau}^{e}(L)$ within the exact calculation as function of the separation length between the walls for different values of the inverse Debye lengths and permittivities of the two liquids. For this plot we consider $\sigma_{1}^{0}$ and $\sigma_{2}^{L}$ to be positive and $\sigma_{1}^{L}$ and $\sigma_{2}^{0}$ to be negative, a case that is not considered in Fig. 3. As one can see, $\omega_{\tau}^{e}(L)$ always shows at least a maximum, the position of which shifts to larger separations with increasing inverse Debye length $\kappa_{1}$ of medium " 1 " or decreasing inverse Debye length $\kappa_{2}$ of medium " 2 ". With decreasing relative permittivity $\varepsilon_{r, 1}$ of medium " 1 ", $\omega_{\tau}^{e}(L)$ can show a single minimum whereas with increasing relative permittivity $\varepsilon_{r, 2}$ of medium " 2 ", it can show a minimum and an additional maximum. Please note that while varying the quantities of medium "1", i.e. in the left panels of Fig. 4 the horizontal axis is 


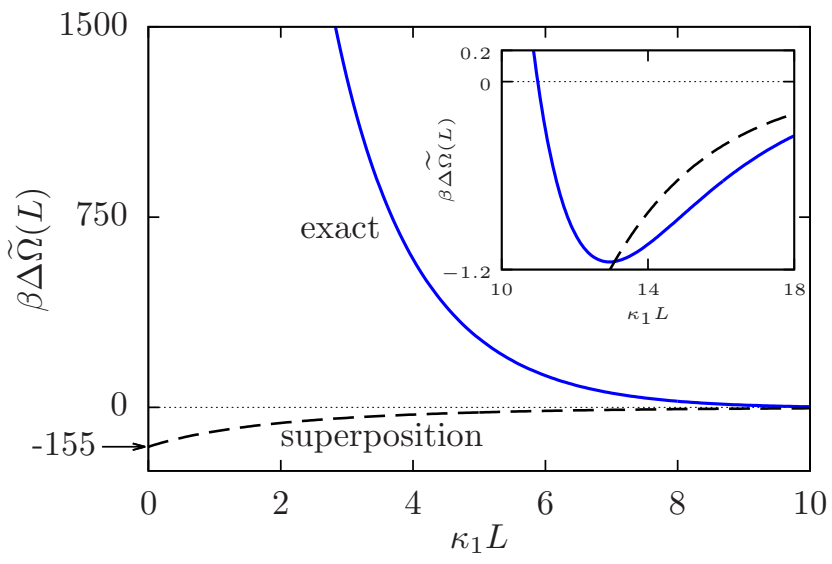

FIG. 5. Total interaction energy $\Delta \widetilde{\Omega}(L)\left(=\omega_{\gamma, 1}(L) A_{1}+\omega_{\gamma, 2}(L) A_{2}+\right.$ $\left.\omega_{\tau}(L) \ell\right)$ between the two surfaces in the units of $1 / \beta$ as a function of the scaled separation $\kappa_{1} L$ for a system with $\sigma_{1}^{0}=0.001 \mathrm{e} / \mathrm{nm}^{2}, \sigma_{1}^{L}=$ $-0.1 \mathrm{e} / \mathrm{nm}^{2}, \sigma_{2}^{0}=0.0001 \mathrm{e} / \mathrm{nm}^{2}, \sigma_{2}^{L}=-0.01 \mathrm{e} / \mathrm{nm}^{2}, \kappa_{1}=0.1 \mathrm{~nm}^{-1}$, $\kappa_{2}=0.03 \mathrm{~nm}^{-1}, \varepsilon_{r, 1}=80, \varepsilon_{r, 2}=2, \beta e \Psi_{D}=1, \ell_{B}=55.7 \mathrm{~nm}$, $A_{1} \approx 7600 \mathrm{~nm}, A_{2} \approx 22000 \mathrm{~nm}$, and $\ell \approx 240 \mathrm{~nm}$. The total effective surface areas $A_{i}$ and the total effective length $\ell$ of the three-phase contact lines are calculated as explained in the main text for particles of radii $R=100 \mathrm{~nm}$. As one can see, whereas the superposition approximation (black dashed line) predicts a deep minimum at vanishing separation between the surfaces leading to aggregation, the exact result (blue solid line) is mostly positive with a shallow minimum (see the inset) at around $\kappa_{1} L \approx 13$ and thus, rules out the possibility of aggregation.

scaled with $\kappa_{2}=0.03 \mathrm{~nm}^{-1}$. Therefore, a maximum even at $\kappa_{2} L \approx 0.3$ corresponds to $L \approx 10 \mathrm{~nm}$, which is well above the molecular dimension.

It is worth mentioning that the corresponding expressions for the identical particles given in Ref. [20] can be recovered from Eqs. (10)-13) by setting $\sigma_{1}^{0}=\sigma_{1}^{L}, \sigma_{2}^{0}=\sigma_{2}^{L}$, and $L=2 L^{\prime}$ where $L^{\prime}$ is the separation between the identically charged walls. This last transformation is needed as the walls in Ref. [20] were considered to be separated by a distance of $2 L$ instead of $L$.

At this point it is natural to ask whether the differences between the exact and the superposition calculations are significant and whether the line part of the interaction can play any significant role compared to the surface contributions for real experimental setups. To answer the first question, we plot in Fig. 5 the total interaction energy $\Delta \widetilde{\Omega}(L)=\omega_{\gamma, 1}(L) A_{1}+$ $\omega_{\gamma, 2}(L) A_{2}+\omega_{\tau}(L) \ell$ between two surfaces in the units of $1 / \beta$ as a function of the separation distance $\kappa_{1} L$ for a typical experimental system with $\sigma_{1}^{0}=0.001 \mathrm{e} / \mathrm{nm}^{2}, \sigma_{1}^{L}=-0.1 \mathrm{e} / \mathrm{nm}^{2}$, $\sigma_{2}^{0}=0.0001 \mathrm{e} / \mathrm{nm}^{2}, \sigma_{2}^{L}=-0.01 \mathrm{e} / \mathrm{nm}^{2}, \kappa_{1}=0.1 \mathrm{~nm}^{-1}$, $\kappa_{2}=0.03 \mathrm{~nm}^{-1}, \varepsilon_{r, 1}=80, \varepsilon_{r, 2}=2, \beta e \Psi_{D}=1, \ell_{B}=55.7 \mathrm{~nm}$, $A_{1} \approx 7600 \mathrm{~nm}^{2}, A_{2} \approx 22000 \mathrm{~nm}^{2}$, and $\ell \approx 240 \mathrm{~nm}$. The values of the effective areas $A_{i}=4\left(2 \kappa_{i}^{-1} R-\kappa_{i}^{-2}\right)$ and of the effective length of the three-phase contact line $\ell=\sqrt{A_{1}}+\sqrt{A_{2}}$ correspond to rough estimates for spherical particles of radii $R=100 \mathrm{~nm}$. These estimates are obtained by noting that the interaction between two colloidal spheres trapped at the interface is essentially given by the interaction between portions of facing caps of height $\kappa_{i}^{-1}$ inside medium $i$, so that, by ignoring irrelevant factors of order unity, $A_{i}$ corresponds to the cap surface area in medium $i$ and $\ell$ to the averaged length of the intersections between the caps and the interface. Note that the effective area $A_{2}$ is larger than $A_{1}$ because the inverse Debye length $\kappa_{2}$ in medium " 2 " is smaller than $\kappa_{1}$ in medium "1". From Fig. 5] one infers that the interaction within the superposition calculation is attractive everywhere with a deep minimum $\left(\approx-155 k_{B} T\right)$ at $\kappa_{1} L=0$. Therefore, according to the superposition calculations, the two particles will stick to each other. However, the exact calculations predict a strong repulsive interaction between the particles with a shallow minimum $\left(\approx-1 k_{B} T\right)$ at separation $\kappa_{1} L \approx 13$ which cannot lead to aggregation of the particles. Thus, depending upon the parameters, the results within the two calculations can lead to completely different qualitative behavior. It is important to understand that the surface contributions to the total interaction energy $\beta \Delta \widetilde{\Omega}(L)$ scale with the square of the radii of the particles whereas the line part scales linearly with the radii. Consequently, for bigger particles, the surfaces parts dominate over the line part. However, for relatively small particles, the line part can easily become comparable to the surface parts to alter both the depth and the position of the minimum of the total interaction energy. For example, if one considers a system with $\sigma_{1}^{0}=0.02 \mathrm{e} / \mathrm{nm}^{2}, \sigma_{1}^{L}=-0.03 \mathrm{e} / \mathrm{nm}^{2}$, $\sigma_{2}^{0}=-0.0004 \mathrm{e} / \mathrm{nm}^{2}, \sigma_{2}^{L}=0.0002 \mathrm{e} / \mathrm{nm}^{2}, \kappa_{1}=0.1 \mathrm{~nm}^{-1}$, $\kappa_{2}=0.01 \mathrm{~nm}^{-1}, \varepsilon_{r, 1}=80, \varepsilon_{r, 2}=2, \beta e \Psi_{D}=1, \ell_{B}=55.7 \mathrm{~nm}$, and $R=150 \mathrm{~nm}$, the minimum of the interaction energy is around $\kappa_{1} L \approx 6.3$ with a depth of $\approx-57 k_{B} T$ when disregarding the line interaction whereas it becomes significantly deeper $\left(\approx-99 k_{B} T\right)$ and shifts to $\kappa_{1} L \approx 1.2$ when including the line contribution. Please note that the surface interaction in the more polar medium decays faster than the one in the less polar medium and the line interaction. As a result, at relatively large separations, the line part competes with the surface interaction in the less polar phase which is weak due to small surface charges in the oil phase.

Although the failure of the superposition approximation is not unexpected at short separations, we would like to emphasize that the main purpose of this study is to provide exact expressions for the interaction energies valid at short separations between particles and to asses the error of the superposition approximation in this limit. Certainly the flat wall assumption fails at large separations between spherical particles, but the factor of " 2 " discrepancies in this limit for all the three interaction contributions are indeed surprising: For two flat surfaces one usually assumes the superposition approximation to work well at large separations as the electrostatic potential is screened in both the fluid phases, and the influence of each surface on the other is expected to vanish exponentially with increasing separation distance. The presence of the fluid interface or the heterogeneities of charge densities on each surfaces cannot be the reason for the difference since we obtain the factor of " 2 " mismatch for the surface interaction energies which are independent of the interface or the surface charge heterogeneity. An open question for future work is the influence of finite particle radii on the quantitative mismatch. 


\section{Wall-fluid(s) and fluid-fluid interactions}

Here we discuss the remaining $L$-independent energy contributions to Eq. (6) which, apart from the bulk contributions $\Omega_{b, i}$, stem from the wall-fluid(s) interactions or from the fluidfluid interactions. The bulk contribution $\Omega_{b, i}$ is independent of the electrostatic potential inside the system and therefore, are the same $\left(-2 I_{i} / \beta\right)$ within both the exact and the superposition calculations. Surface tensions acting between the walls placed at $z=0$ or $z=L$ and medium " 1 " are given by $\gamma_{1}^{0}=\frac{\left(\sigma_{1}^{0}\right)^{2}}{2 \kappa_{1} \varepsilon_{1}}$ or $\gamma_{1}^{L}=\frac{\left(\sigma_{1}^{L}\right)^{2}}{2 \kappa_{1} \varepsilon_{1}}$, respectively, within both the calculations. However, for medium " 2 ", the surface tensions acting between the wall at $z=0$ and the fluid medium "2" within the exact calculations is given by $\gamma_{2}^{0, e}=\frac{\left(\sigma_{2}^{0}\right)^{2}}{2 \kappa_{2} \varepsilon_{2}}+\sigma_{2}^{0} \Psi_{D}$, whereas within the superposition approximation it is given by $\gamma_{2}^{0, s}=\frac{\left(\sigma_{2}^{0}\right)^{2}}{2 \kappa_{2} \varepsilon_{2}}+\frac{3}{2} \sigma_{2}^{0} \Psi_{D}$. These expressions remain the same for $\gamma_{2}^{L}$ within the two calculations albeit with $\sigma_{2}^{L}$ in place of $\sigma_{2}^{0}$. The interfacial tension $\gamma_{1,2}$ acting between the two fluids within the two calculation schemes also differ from each other. Whereas the exact calculations give $\gamma_{1,2}^{e}=-\frac{\kappa_{1} \kappa_{2} \varepsilon_{1} \varepsilon_{2} \Psi_{D}^{2}}{2\left(\kappa_{1} \varepsilon_{1}+\kappa_{2} \varepsilon_{2}\right)}$, superposition calculations lead to an exactly doubled value: $\gamma_{1,2}^{S}=-\frac{\kappa_{1} \kappa_{2} \varepsilon_{1} \varepsilon_{2} \Psi_{D}^{2}}{\kappa_{1} \varepsilon_{1}+\kappa_{2} \varepsilon_{2}}$. The line tension acting at the three-phase contact line at $z=0$ due to an interaction of the wall with the two fluids is given within the exact calculations $\left(\tau^{0, e}\right)$ and the superposition approximation $\left(\tau^{0, s}\right)$ by:

$$
\tau^{0, e}=\frac{\kappa_{2} \varepsilon_{2} \Psi_{D}}{\kappa_{1} \varepsilon_{1}+\kappa_{2} \varepsilon_{2}}\left(\frac{\sigma_{1}^{0}}{\kappa_{1}}-\frac{\kappa_{1} \varepsilon_{1}}{\kappa_{2} \varepsilon_{2}} \frac{\sigma_{2}^{0}}{\kappa_{2}}\right)+\frac{1}{\pi} \int_{0}^{\infty} \frac{d q}{p_{1}(q) \varepsilon_{1}+p_{2}(q) \varepsilon_{2}}\left(\frac{2 \sigma_{1}^{0} \sigma_{2}^{0}}{p_{1}(q) p_{2}(q)}-\frac{p_{2}(q) \varepsilon_{2}}{p_{1}(q) \varepsilon_{1}}\left(\frac{\sigma_{1}^{0}}{p_{1}(q)}\right)^{2}-\frac{p_{1}(q) \varepsilon_{1}}{p_{2}(q) \varepsilon_{2}}\left(\frac{\sigma_{2}^{0}}{p_{2}(q)}\right)^{2}\right),
$$

and

$$
\tau^{0, s}=\frac{3}{2} \frac{\kappa_{2} \varepsilon_{2} \Psi_{D}}{\kappa_{1} \varepsilon_{1}+\kappa_{2} \varepsilon_{2}}\left(\frac{\sigma_{1}^{0}}{\kappa_{1}}-\frac{\kappa_{1} \varepsilon_{1}}{\kappa_{2} \varepsilon_{2}} \frac{\sigma_{2}^{0}}{\kappa_{2}}\right)+\frac{1}{\pi} \int_{0}^{\infty} \frac{d q}{p_{1}(q) \varepsilon_{1}+p_{2}(q) \varepsilon_{2}}\left(\frac{2 \sigma_{1}^{0} \sigma_{2}^{0}}{p_{1}(q) p_{2}(q)}-\frac{p_{2}(q) \varepsilon_{2}}{p_{1}(q) \varepsilon_{1}}\left(\frac{\sigma_{1}^{0}}{p_{1}(q)}\right)^{2}-\frac{p_{1}(q) \varepsilon_{1}}{p_{2}(q) \varepsilon_{2}}\left(\frac{\sigma_{2}^{0}}{p_{2}(q)}\right)^{2}\right),
$$

respectively. Corresponding expressions for $\tau^{L, e}$ and $\tau^{L, s}$ can be obtained by replacing $\sigma_{i}^{0}$ with $\sigma_{i}^{L}$ in the two expressions. Although the integral term is the same in both expressions, clearly $\tau^{0, e} \neq \tau^{0, s}$ due to an additional prefactor of $\frac{3}{2}$ present in the first term of Eq. (15). This is related to the fact that the exact electrostatic potential $\Psi_{i}^{e}(x, z)$ in Eq. (7) reduces to the single wall potential $\Psi_{i}^{\sin }(x, z)$ in Eq. (8) in the limit $L \rightarrow$ $\infty$ with $\sigma_{2}^{0}=\sigma_{2}^{L}=0$, whereas the superposition potential $\Psi_{i}^{s}(x, z)$ in Eq. (9) does not.

\section{CONCLUSIONS}

In summary, by using a classical DFT approach, we have analyzed the electrostatic interaction between two unequally charged parallel walls in contact with two immiscible liquids. Within the framework of a linearized PB mean-field theory, we have derived analytical expressions for the electrostatic potential distribution inside the system both within and by going beyond the linear superposition approximation. Then these potentials are used to calculate the surface and line interaction energy densities between the walls. As an important finding we see that both the surface and the line interaction can vary monotonically and non-monotonically and in most cases, the superposition approximation fails to predict the correct variations at short separations. Moreover, contrary to common assumption, the superposition approximation can be quantitatively as well as qualitatively incorrect even at large separations. Analytical expressions are also provided for other interaction parameters of the system, i.e., the surface tensions, the line tensions and the fluid-fluid interfacial tension. It turns out that all these constant (independent of the separation distance between the walls) interaction parameters also differ more or less within the two calculation schemes. The system under consideration is expected to mimic the interaction between two dissimilar colloids trapped at an electrolyte interface with a surface to surface separation distance small compared to the radii of the particles. Not only that, our general study can also be applied to other situations like the interaction between two Janus colloids in the bulk or at an interface or a single Janus particle approaching a solid surface [39].
[1] W. Ramsden, Proc. R. Soc. London 72, 156 (1903).

[2] S. U. Pickering, J. Chem. Soc. Trans. 91, 2001 (1907).

[3] B. P. Binks and T. S. Horozov, Colloidal Particles at Liquid Interfaces (Cambridge University Press, Cambridge, 2006).
[4] M. A. Ray, N. Shewmon, S. Bhawalkar, L. Jia, Y. Yang, and E. S. Daniels, Langmuir, 25, 7265 (2009).

[5] L. Isa, I. Buttinoni, M. A. Fernandez-Rodriguez, and S. A. Vasudevan, Eur. Phys. Lett., 119, 26001 (2017). 
[6] S. Abend, N. Bonnke, U. Gutschner, and G. Lagaly, Colloid Polym. Sci. 276, 730 (1998).

[7] Y. Mao and D. J. McClements, Food Hydrocolloids 25, 1201 (2011).

[8] Y. Mao and D. J. McClements, Food Hydrocolloids 27, 80 (2012).

[9] A. D. Dinsmore, M. F. Hsu, M. G. Nikolaides, M. Márquez, A. R. Bausch, and D. A. Weitz, Science 298, 1006 (2002).

[10] M. Li, R. L. Harbron, J. V. M. Weaver, B. P. Binks, and S. Mann, Nat. Chem. 5, 529 (2013).

[11] S. Abend and G. Lagaly, Clay Miner. 36, 557 (2001).

[12] B. P. Binks, W. Liu, and J. A. Rodrigues, Langmuir 24, 4443 (2008).

[13] T. Nallamilli, E. Mani, and M. G. Basavaraj, Langmuir 30, 9336 (2014).

[14] T. Nallamilli, B. P. Binks, E. Mani, and M. G. Basavaraj, Langmuir 31, 11200 (2015).

[15] H. Wang, V. Singh, and S. H. Behrens, J. Phys. Chem. Lett. 3, 2986 (2012).

[16] A. Majee, M. Bier, and R. Podgornik, Soft Matter 14, 985 (2018).

[17] P. Pieranski, Phys. Rev. Lett. 45, 569 (1980).

[18] A. J. Hurd, J. Phys. A 18, L1055 (1985).

[19] Z. Lian, J. Chem. Phys. 145, 014901 (2016).

[20] A. Majee, M. Bier, and S. Dietrich, J. Chem. Phys. 140, 164906 (2014).

[21] A. Majee, M. Bier, and S. Dietrich, J. Chem. Phys. 145, 064707 (2016).

[22] R. Aveyard, B. P. Binks, and J. H. Clint, Adv. Colloid Interface Sci. 100, 503 (2003).

[23] F. Reincke, S. G. Hickey, W. K. Kegel, and D. Vanmaekelbergh, Angew. Chem. Int. Ed. 43, 458 (2004).
[24] K. Masschaele, B. J. Park, E. M. Furst, J. Fransaer, and J. Vermant, Phys. Rev. Lett. 105, 048303 (2010).

[25] P. V. Petkov, K. D. Danov, and P. A. Kralchevsky, Langmuir 30, 2768 (2014).

[26] P. V. Petkov, K. D. Danov, and P. A. Kralchevsky, J. Colloid Interface Sci. 462, 223 (2016).

[27] P. A. Kralchevsky, K. D. Danov, and P. V. Petkov, Phil. Trans. R. Soc. A 374, 20150130 (2016).

[28] S. Uppapalli and H. Zhao, Soft Matter 10, 4555 (2014).

[29] S. D. C. Pushpam, M. G. Basavaraj, and E. Mani, Phys. Rev. E 92, 052314 (2015).

[30] G. V. Bossa, J. R. K. Bohinc, and S. May, Soft Matter 12, 4229 (2016).

[31] R. Aveyard, J. H. Clint, D. Nees, and V. N. Paunov, Langmuir 16, 1969 (2000).

[32] R. Aveyard, B. P. Binks, J. H. Clint, P. D. I. Fletcher, T. S. Horozov, B. Neumann, V. N. Paunov, J. Annesley, S. W. Botchway, D. Nees, A. W. Parker, A. D. Ward, and A. N. Burgess, Phys. Rev. Lett. 88, 246102 (2002).

[33] B. J. Park, J. P. Pantina, E. M. Furst, M. Oettel, S. Reynaert, and J. Vermant, Langmuir 24, 1686 (2008).

[34] P. Gao, X. C. Xing, Y. Li, T. Ngai, and F. Jin, Sci. Rep. 4, 4778 (2014).

[35] C. P. Kelleher, A. Wang, G. I. Guerrero-García, A. D. Hollingsworth, R. E. Guerra, B. J. Krishnatreya, D. G. Grier, V. N. Manoharan, P. M. Chaikin, Phys. Rev. E 92, 062306 (2015).

[36] M. Bier, A. Gambassi, and S. Dietrich, J. Chem. Phys. 137, 034504 (2012).

[37] T. Schmetzer, Electrostatic interaction between non-identical charged particles at an electrolyte interface, B. Sc. thesis, University of Stuttgart (2017), see arXiv:1710.05415

[38] V. S. Bagotsky, Fundamentals of Electrochemistry (Wiley, Hoboken, NJ, 2006).

[39] A. Rashidi and C. L. Wirth, J. Chem. Phys. 147, 224906 (2017). 